\title{
Quasiparticle transport equation with collision delay. II. Microscopic Theory
}

\author{
Václav Špička and Pavel Lipavský \\ Institute of Physics, Academy of Sciences, Cukrovarnická 10, 16200 Praha 6, Czech Republic \\ Klaus Morawetz \\ Max-Planck Gesellschaft, Arbeitgruppe "Theoretische Vielteilchenphysik" an der Universität Rostock, D-18055 Rostock, \\ Germany \\ (August 28, 2018)
}

\begin{abstract}
For a system of non-interacting electrons scattered by neutral impurities, we derive a modified Boltzmann equation that includes quasiparticle and virial corrections. We start from quasiclassical transport equation for non-equilibrium Green's functions and apply limit of small scattering rates. Resulting transport equation for quasiparticles has gradient corrections to scattering integrals. These gradient corrections are rearranged into a form characteristic for virial corrections.
\end{abstract}

\section{INTRODUCTION}

In the first paper of this seriest (referred as Paper I), we have discussed interplay of quasiparticle and virial corrections for scattering by neutral impurities with resonant levels. We have found that both corrections are of the same order and tend to mutually compensate. Accordingly, one should either include both kinds of corrections or neglect both of them. Separate quasiparticle or separate virial corrections lead to overestimates of impurity effect on basic physical quantities like dc conductivity or screening length.

In Paper I we have used an intuitive modification of the Boltzmann equation (BE), Eq. (I-39) [Eq. (39) of Paper I]. Long time experience with quasiparticle corrections shows that intuitive approaches whatever convincing are far from to be reliable as the wave-function renormalization factor often emerges in an unexpected way. To become trustworthy, Eq. (I-39) has to be recovered from quantum statistics in a very systematic manner. This is the aim of this paper.

The intuitive modification of the BE we would like to arrive to reads

$$
\begin{aligned}
\frac{\partial f}{\partial t} & +z \frac{k}{m} \frac{\partial f}{\partial r}-\frac{\partial \phi}{\partial r} \frac{\partial f}{\partial k}=-\frac{f}{\tau} \\
& +\frac{1}{\tau} \frac{2 \pi^{2}}{k^{2}} \int \frac{d p}{(2 \pi)^{3}} \delta(|p|-|k|) f\left(p, r, t-\Delta_{t}\right) .
\end{aligned}
$$

This is equation (I-39). It applies to system of noninteracting electrons scattered by point impurities with resonant levels. Here, the quasiparticle distribution $f$ is a function of momentum $k$, coordinate $r$ and time $t$. Lifetime $\tau$, collision delay $\Delta_{t}$ and wave-function renormalization $z$ are functions of momentum, potential $\phi$ depends on coordinate and time. The collision delay $\Delta_{t}$ (given by the energy derivative of the phase shift) makes the scattering-in integral non-local in time. This nonlocality represents virial corrections. The quasiparticle corrections are covered by the wave-function renormalization $z$.
So far, quasiparticle and virial corrections have been studied only separately using different theoretical tools. Now we briefly review previous studies to identify which tool is better suited for unified theory.

\section{A. Virial corrections}

A need to derive virial corrections to a quantum transport equation has been felt for a while. The progress achieved in this direction can be represented by Snider's equation.23 Using the BBGKY hierarchy, Snider has derived a quantum transport equation for the reduced density matrix (Wigner's distribution). Snider's equation is sufficiently general to describe various corrections be yond the BE, however, as noticed by Laloë and Mullin,t all corrections enter the scattering integral, while quasiparticle corrections should appear rather as corrections to drift of single-particle excitations.

Snider's equations includes another item alien to the quasiparticle picture: the reduced density matrix. When the quasiparticle corrections are in game, the transport equation should deal with the quasiparticle distribution not with the reduced density matrix. In the reduced density matrix, contributions from free excitations (quasiparticles) and from correlations (off-shell motion during dressing processes) are mixed together. The drift of free excitations can be described by simple quasiclassical trajectories while correlations require quantum mechanical treatment. To be able to make efficient approximations, these two types of motion has to be separated. So far, the theory of transport based on the reduced density matrix is missing a tool that would furnish us with such a separation.

\section{B. Quasiparticle corrections}

The second group of papers is a large variety of studies recovering the Boltzmann equation with quasiparticle corrections in the form visualized by Landau, e.g. 
Refs. 5 14. On the other hand, none of these studies touches virial corrections. One expects that the virial corrections should emerge from systematic quantumstatistical approaches to the Landau theory provided one does not loose them making unjustified approximations.

¿From the intuitive modification of the BE (1), we can identify three groups of approximations we have to avoid:

- Most of authors limit their attention to the scattering described within the Born approximation (as we have also done in Ref. 14). The corresponding T-matrix is just an impurity potential that does not cause any collision delay. Similarly, the selfconsistent Puff-Whitefield approximation of the electron-phonon scattering (the Migdal approximation with non-self-consistent phonons) studied by Prange and Kadanoff, gives no virial corrections since the interaction vertex has no internal electron dynamics like the T-matrix within the Born approximation.

- Prange and Sach 10 have studied the electronelectron and electron-phonon scattering within the fully self-consistent single-loop approximation. The screened Coulomb interaction is in general a complex function of energy so that it yields some kind of virial corrections. Prange and Sachs have used, however, the static approximation within which the screened Coulomb potential looses energy dependence and becomes real. In this way, the virial corrections are lost.

- Danielewic211 and Botermans and Malfliet12 have used the two-particle T-matrix for nucleon-nucleon interaction that definitely includes virial corrections, however, they have neglected gradient contributions to the scattering integral. From the nonlocal character of the scattering integral in the intuitive BE (1), one can see that the virial corrections are proportional to gradients of the quasiparticle distribution, see (2). Neglecting gradient contributions one looses the virial corrections.

In all the three cases, one can go beyond these approximations. To recover Eq. (11), we have to use the T-matrix for the impurity scattering and keep the gradient corrections to scattering integrals. Similar treatment has lead to virial corrections for two-particle scattering.15

\section{Gradient corrections to scattering integrals}

The gradient corrections to the scattering integral are of central importance in our treatment. In the intuitive $\mathrm{BE}$ (1), the only gradient contributions to the scattering integral come from the virial corrections,

$$
\frac{\partial f}{\partial t}+z \frac{k}{m} \frac{\partial f}{\partial r}-\frac{\partial \phi}{\partial r} \frac{\partial f}{\partial k}=-\frac{f}{\tau}
$$

$$
\begin{aligned}
& +\frac{1}{\tau} \frac{2 \pi^{2}}{k^{2}} \int \frac{d p}{(2 \pi)^{3}} \delta(|p|-|k|) f(p, r, t) \\
& -\frac{1}{\tau} \frac{2 \pi^{2}}{k^{2}} \int \frac{d p}{(2 \pi)^{3}} \delta(|p|-|k|) \Delta_{t} \frac{\partial}{\partial t} f(p, r, t) .
\end{aligned}
$$

Accordingly, we expect that gradient corrections resulting within the standard approaches to the BE are just the virial corrections. Our plan is to derive gradient corrections from the quasiclassical limit of the quantumstatistical transport equation and rearrange them into the last term of Eq. (2).

Gradient corrections to scattering integrals have been already studied by many authors, the most extensive studies were devoted to the effect of electric field on collisions. Starting from Barker 16 , the field effect on scattering has been discussed for number of scattering mechanisms within various approaches which include the superoperator mrojection technique, Refs. 16,17, the Levinson equation 18 derived from the BBGKY hierarchy, Ref. 19, and non-equilibrium Green's functions, Refs. 2025.

It is important to note that these different approaches resulted in contradictory predictions. Let us focus on the (linear) gradient correction to the scattering integral which we claim to be equal to the virial correction [the last term in the r.h.s of (2)]. We will assume only selfenergies in the Born or Puff-Whitefield approximation so that the virial corrections are absent, i.e., we expect the gradient corrections to be absent, too. Surprisingly, this is not true about gradient corrections one finds in print. One can split all papers into three groups:

1. In Refs. 16, 17, 19,24, linear gradient corrections were found.

2. In Ref. 25 linear gradient corrections were found but with opposite sign than in the first group.

3. In Refs. 2023 no linear gradient corrections were found.

Unless we will understand why the gradient corrections appear for the Born or Puff-Whitefield approximation and why there are contradictory predictions from alternative approaches, we can hardly use the gradient corrections as a start line of our approach to the virial corrections.

To identify the origin of the three contradictory predictions, let us inspect what kind of the transport equation is specific for each group. Although various approaches have been used by authors, we will describe all the three groups within a common dialect of Green's functions.

\section{Integro-differential transport equation for the reduced density matrix}

The integro-differential transport equation for the reduced density matrix is obtained by the Generalized Kadanoff and Baym (GKB) ansatz 24 implemented in the 
time diagonal of the integro-differential Kadanoff and Baym (KB) equation $\bar{E}$. ¿From the GKB ansatz, time argument $t$ of the reduced density matrix results retarded compared to the time argument $T$ of the scattering integral, i.e. $t<T$. In the integro-differential equation, it is natural to identify the instant of the collision with $T$. Using the linear expansion $\rho(t)=\rho(T)+\frac{\partial \rho}{\partial t}(t-T)$ one then obtains a Boltzmann-like scattering integral from $\rho(T)$ and gradient corrections from $\frac{\partial \rho}{\partial t}(t-T)$.

\section{Integral transport equation for the reduced density matrix}

The integral transport equation for the reduced density matrix is obtained by the GKB ansatz implemented in the time diagonal of the integral GKB equation 26. In the integral equation one has a freedom to identify the instant of the collision with $t$ and gradient corrections emerge from matching of the scattering integral with the subsequent propagation. The opposite signs found in groups 1 and 2 thus follow from the fact that in group 1 authors extrapolate along the initial state of the collision while in group 2 authors extrapolate along the final one.

\section{Integro-differential transport equation for the quasiparticle distribution}

The transport equation for the quasiparticle distribution is obtained by the original KB ansat 5 implemented to the KB equation at the quasiparticle pole. From the $\mathrm{KB}$ ansatz, the time argument of the quasiparticle distribution results identical to the time argument of the scattering integral. This time is naturally identified as an instant of the collision event and no gradient corrections appear.

The contradictory predictions of the gradient corrections were found to follow from different treatments of quasiparticle corrections.27 As shown in Ref. 27, the linear gradient correction to the rate of scattering out is nothing but the time dependence of the wave-function renormalization. In other words, gradient and quasiparticle corrections are linked together. The approach that takes care of quasiparticle features and naturally leads to the Landau-Boltzmann type of transport equation for quasiparticles is the one of the group 3, i.e., based on the KB ansatz. The absence of the gradient corrections for the Born approximation is also in agreement with our expectation.

\section{Non-virial gradient corrections}

Among studies of the gradient corrections that are not devoted to the field effect we vant to point out the paper by Kolomiets and Plyuiko28. In the quasiclassical limit, they have evaluated the scattering integral from the self-energy in the second-order approximation of the electron-electron interaction keeping all gradient terms. Similarly to the spirit of classical virial corrections, they have expressed gradient corrections in terms of effective shifts in space and momentum. The scattering integral they have derived thus reminds the one we are looking for.

In spite of formal similarity, the corrections derived by Kolomiets and Plyuiko are not the virial corrections. Kolomiets and Plyuiko have used a mixed approach implementing the KB ansatz in the time diagonal of the integro-differential form of the GKB equation. These two steps are not compatible as the KB ansatz includes the quasiparticle distribution while the time diagonal (or integral over energy in Wigner's representation) provides drift terms for the reduced density matrix. The gradient contributions they have found are thus a strange form of the quasiparticle corrections.

Again we came to the same moral. If we want to put equality between the virial and the gradient corrections to the scattering integral, we have to work fully in the quasiparticle picture.

\section{E. Presented approach}

Our approach is based on two limits applied to nonequilibrium Green's functions. The first one is the quasiclassical limit that is inevitable for the BE5 The second one is the limit of small scattering rates.14 This limit restricts validity of quasiparticle corrections to weakly renormalized systems. The validity of virial corrections is restricted to the second virial coefficient.

The limit of small scattering rates from the quasiclassical transport equation for non-equilibrium Green's function has been already presented in detail in Ref. 14. In fact, the transport equation for quasiparticles, Eq. (70) of Ref. 14, reduces to the modified BE (11). From this point of view, this paper is just an application of the method derived in Ref. 14. On the other hand, in Ref. 14 authors discussed only simple self-energies on the level of Born approximation which provide quasiparticle corrections but no virial corrections appear. Accordingly, there is no collision delay. Here we use a more complex selfenergy, averaged T-matrix approximation, that results in non-trivial virial corrections similarly as it was done in Ref. 15 for two-particle scattering.

In spite or because of many similar and closely related studies of gradient contributions to transport equations, we feel that we have to start our treatment directly from non-equilibrium Green's functions instead of recalling already achieved results. This is because these treatments differ in seemingly tiny details that become important as soon as one wants to keep trace of gradients and quasiparticle corrections in the same time.

Now we can specify our aim. The precursor of equation (1) is the quasiparticle Boltzmann equation in semicon- 
ductors, Eq. (70) of Ref. 14, which reads

$$
\frac{\partial f}{\partial t}+\frac{\partial \varepsilon}{\partial k} \frac{\partial f}{\partial r}-\frac{\partial \varepsilon}{\partial r} \frac{\partial f}{\partial k}=-z\left(\gamma_{\varepsilon} f-\sigma_{\varepsilon}^{<}\right) .
$$

Except for transport vertex $\sigma^{<}$, all components are determined by retarded self-energy $\sigma^{R}$. First, $\gamma=-2 \operatorname{Im} \sigma^{R}$ provides inverse lifetime, $\frac{1}{\tau}=z \gamma_{\varepsilon}$. Second, the quasiparticle energy is given by $\varepsilon=\frac{k^{2}}{m}+\phi+\operatorname{Re} \sigma_{\varepsilon}^{R}$. Note that unlike in Paper I, $\varepsilon$ includes the potential $\phi$. We made this change of convention to comply with the convention of Ref. 14 .

It will be easy to show that for homogeneously distributed point impurities the velocity simplifies to the form in (1), $\frac{\partial \varepsilon}{\partial k}=z \frac{k}{m}$, and similarly do the force, $\frac{\partial \varepsilon}{\partial r}=\frac{\partial \phi}{\partial r}$. Apparently, quasiparticle corrections are explicitly present in equation (3). The viral corrections, if present, are hidden in the transport vertex $\sigma^{<}$. The focus of our interest thus will be to show that the transport vertex can be rearranged to the form of the scattering-in integral from (2),

$$
\begin{aligned}
z \sigma_{\varepsilon}^{<} & =\frac{1}{\tau} \frac{2 \pi^{2}}{k^{2}} \int \frac{d p}{(2 \pi)^{3}} \delta(|p|-|k|) f(p, r, t) \\
& -\frac{1}{\tau} \frac{2 \pi^{2}}{k^{2}} \int \frac{d p}{(2 \pi)^{3}} \delta(|p|-|k|) \Delta_{t} \frac{\partial}{\partial t} f(p, r, t) .
\end{aligned}
$$

\section{F. Content}

Paper is organized as follows. In Sec. II, we briefly review the method of Ref. 14. We introduce the nonequilibrium Green's functions, the quasiclassical limit, and the limit of small scattering rates. With help of these tools the Boltzmann-like transport equation is derived. In Sec. III, the self-energy and the transport vertex are specified within the averaged T-matrix approximation. The most essential part of our paper is in Sec. IV, where we evaluate the scattering integral including its gradient corrections. In Sec. V, these gradient corrections are rearranged into a form that is identical to the one intuitively expected and equation (11) is recovered. In Sec. VI, singleelectron observables are valuated. Electron density and current are discussed in detail. We also discuss the density of energy. Sec. VII includes summary. In Appendix we discuss relation between virial and quasiparticle corrections.

\section{TRANSPORT EQUATION}

Our focus of interest is the transport vertex $\sigma^{<}$. The treatment of the transport vertex, however, is intimately connected to the transport equation itself. Therefore we find it profitable to briefly review derivation of equation (3). This also gives us a room to introduce a necessary set of equations for non-equilibrium Green's functions and their relations to components of equation (3).

\section{A. Generalized Kadanoff and Baym equation}

Our starting point is the generalized Kadanoff and Baym equatione

$$
G^{<}=G^{R} \Sigma^{<} G^{A}
$$

that is accompanied by the Dyson equation for retarded (advanced) propagator 26

$$
\left(G_{0}^{-1}-\Sigma^{R, A}\right) G^{R, A}=1,
$$

where the inverse free-particle propagator reads

$$
G_{0}^{-1}(1,2)=\left(\frac{\partial}{\partial t_{1}}+\epsilon\left(\frac{1}{i} \frac{\partial}{\partial x_{1}}\right)-\phi\left(x_{1}, t_{1}\right)\right) \delta(1-2) .
$$

Here, numbers are cumulative variables $1 \equiv\left(t_{1}, x_{1}\right)$, time and space. Matrix products include integrations over time and space, $C=A B$ means $C(1,2)=$ $\int d t_{3} d x_{3} A(1,3) B(3,2)$. The $\epsilon$ is the free-electron kinetic energy and $\phi$ is a potential. Our sign convention for the correlation function $G^{<}$is

$$
G^{<}(1,2)=\operatorname{Tr}\left(\hat{\rho} \psi^{\dagger}(2) \psi(1)\right),
$$

where $\hat{\rho}$ is the grand-canonical averaging operator, and $\psi^{\dagger}$ and $\psi$ are creation and anihilation operators, respectively.

Equations (5-8) are general identities and definitions. The particular physical content of these equations is specified by self-energy $\Sigma^{R, A}$ and transport vertex $\Sigma^{<}$. The transport equation (3) follows from set (5-7) with no regards to a particular form of self-energy, except that the scattering rate connected to $\Sigma^{R, A}$ and $\Sigma^{<}$is supposed to be small in a sense specified below. Moreover, perturbations in the system of electrons are supposed to be smooth in time and space so that corresponding gradients are also small. Thus we leave specification of the self-energy for next section, and focus on the quasiclassical limit (small gradients) and limit of small scattering rates of equations (5-7).

\section{B. Wigner's representation}

In the quasiclassical limit, all operators are conveniently described in Wigner's mixed representation

$$
\begin{aligned}
a(\omega, k, r, t) & =\int d \tau d y \mathrm{e}^{i \omega \tau-i k y} \\
& \times A(t+\tau / 2, r+y / 2, t-\tau / 2, r-y / 2) .
\end{aligned}
$$

We use convention that lowercase denotes Wigner's transform of operators denoted by uppercase. This convention is in agreement with the convention used in $\mathrm{Pa}$ per I. Indeed, site-diagonal operators used in Paper I (like 
$\Sigma^{R, A,<}, V$ and $\left.T^{R, A}\right)$ in Wigner's representation are momentum independent and equal to their local elements $\sigma^{R, A,<}, v$ and $t^{R, A}$ introduced in Paper I.

The transformation (9) mixes together left and right arguments of the function $A$, therefore it complicates matrix products. Keeping only the first gradients in time and coordinates, the matrix product $C=A B$ in the mixed representation reads

$$
c=a b+\frac{i}{2}[a, b]
$$

where the rectangular brackets denotes Poisson's brackets

$$
[a, b](\omega, k, r, t)=\frac{\partial a}{\partial \omega} \frac{\partial b}{\partial t}-\frac{\partial a}{\partial t} \frac{\partial b}{\partial \omega}-\frac{\partial a}{\partial k} \frac{\partial b}{\partial r}+\frac{\partial a}{\partial r} \frac{\partial b}{\partial k} .
$$

\section{Propagation of single quasiparticle}

In the quasiclassical limit, one restricts the assumed fields $\phi$ to those that vary slowly in time and space. In this case, the field is expected to have a pronounced effect only on long trajectories of electrons. Such long trajectories necessarily include number of collisions with impurities. A propagation between subsequent collisions and individual collisions themselves happen on a very small time and space scale, thus they should be nearly the same as in a homogeneous and stationary potential, i.e., in the absence of the field. There might be also a small effect of the field on these microscopic scales, however, this field effect can be handled as a correction linear in gradients of the field.

Propagators $G^{R, A}$ describe a motion between collisions and also internal dynamics of collisions. Thus we have to find them up to linear order of gradients. From the Dyson equation (6) and its alternative form $G^{R, A}\left(G_{0}^{-1}-\Sigma^{R, A}\right)=1$ one finds that propagators are free of gradients 5

$$
g^{R, A}=\frac{1}{\omega-\epsilon-\phi-\sigma^{R, A}} .
$$

Being a complex function,

$$
\sigma^{R, A}=\sigma \mp \frac{i}{2} \gamma
$$

the self-energy describes two kinds of phenomena. Its imaginary part $\frac{1}{2} \gamma$ descries scattering out of the state of momentum $k$. Its real part $\sigma$ renormalizes energy of the single-particle-like state. The renormalized (quasiparticle) energy is given by a position of the pole of $G^{R, A}$ at the real axis

$$
\varepsilon=\epsilon+\phi+\sigma(\varepsilon)
$$

This quasiparticle energy is an ingredient of the quasiparticle BE (3).

In the limit of small scattering rates (small $\gamma$ expansion, $-2 \operatorname{Im} \sigma^{R} \equiv \gamma \rightarrow 0$ ), the pole of the propagator sits close to the real axis. Then one approximates the spectral function

$$
a=-2 \operatorname{Im} g^{R}
$$

by its limiting value

$$
a=2 \pi z \delta(\omega-\varepsilon)+\gamma \operatorname{Re} \frac{1}{(\omega-\epsilon-\phi-i 0)^{2}} .
$$

Within the quasiparticle picture, in the spectral function $a(\omega, k, r, t)$, the $\delta$ function represents a singular contribution from quasiparticle state of momentum $k$ (at point $r$ and time $t$ ). The second term describes various projections of other quasiparticle states with energy $\varepsilon(p, r, t)=\omega$ into momentum $k$, in other words, the second term is an off-pole contribution. Within the quasiparticle picture, these two parts of the spectral function has to be treated separately.

The norm $z$ of the $\delta$ function is the wave-function renormalization

$$
z=\frac{1}{1-\left.\frac{\partial \sigma(\omega)}{\partial \omega}\right|_{\omega=\varepsilon}} .
$$

\section{Completed collisions}

The generalized Kadanoff and Baym equation (5) includes all phenomena we are interested in, but in a hidden form. The simple link between Green's function formulae and intuitive approach to the $\mathrm{BE}$ with virial corrections is obscured by the fact that these two approaches deal with different objects. While the correlation function $G^{<}$includes both, the pole and off-pole, contributions, the quasiparticle distribution is related only to the pole part. To recover the BE equation, one has to separate these two parts.

Within Green's function the pole and off-pole propagation are described by a single transport equation (5). In contrast, a diffusion of particles described by the LHS of the BE applies only to the pole part, while the offpole part seems to be missing. In fact, it is not missing. The off-pole propagation is hidden in scattering integrals and relation between observables and the quasiparticle distribution.

The possibility to move the off-pole propagation into the scattering integral follows from a hierarchy of time and space scales in the system that are inevitable for the theory of Boltzmann type. Diffusion of quasiparticles is well defined on a hydrodynamical scale that includes a large number of impurities and a large number of collisions per particle. On the hydrodynamical scale, there 
is an appreciable effect of the field $\phi$ which may cause a transfer of quasiparticles over long distances. Such a massive changes of the system state is effectively described with help of a differential transport equation that balances drift with dissipation. In contrast, individual collisions happen on a microscopic (local) scale on which the effect of the field $\phi$ is small so that it can either be neglected or included by corrections linear in its gradients. Thus, a subdynamics on the microscopic scale can be integrated through and approximated by effective scattering rates.

Clearly, to recover the $\mathrm{BE}$ we have to separate hydrodynamical and microscopic scales. This separation is equivalent to the separation of the pole and off-pole parts based on a non-equilibrium mpdification of the expansion in small scattering rates.29.14 Alternatively, the separation of pole and off-pole parts of $G^{<}$can be based on the idea of completed collisions. In this paper we focus on the microscopic mechanism of collisions, thus we follow the completed collision approach.

For the purpose of motivation, we assume for a while a homogeneous system that is diagonal in momentum representation. Let us take a look on a time-diagonal element of $G^{<}$, say $G^{<}(t, t ; k)$. In the transport equation (17), the transport vertex $\Sigma^{<}$represents the last collision due to which a wave function of electron gained a component of the momentum $k$. This component can belong either to an asymptotic state that will form new effective quasiparticle state or to the off-pole contribution due to some other state $p$. The asymptotic state is on the energy shell, thus it will live on the time scale of the quasiparticle life time. The off-pole contribution will vanish on the scale of a quasiparticle formation time. The latter is much shorter than the former. To distinguish whether the contribution to $G^{<}(t, t ; k)$ is of pole or off-pole nature, one can monitor a vicinity of the time $t$, including a close future, to figure out which part will survive and which part soon disappears. This procedure corresponds the approach of Fermi golden rule, where one also integrates through a collision into future and uses matching of asymptotic states to identify a product of the completed collision.

In accordance with the causality principle, all time integrals in (17) run only over the left part of the time axis, i.e., for times smaller than $t$. To monitor a close time vicinity, we rearrange the transport equation (17) as

$$
\begin{aligned}
G^{<} & =\frac{1}{2}\left(G^{R}-G^{A}\right) \Sigma^{<} G^{A}-\frac{1}{2} G^{R} \Sigma^{<}\left(G^{R}-G^{A}\right) \\
& +\frac{1}{2} G^{R} \Sigma^{<} G^{R}+\frac{1}{2} G^{A} \Sigma^{<} G^{A} .
\end{aligned}
$$

In the added term

$$
\Xi^{<}=\frac{1}{2} G^{R} \Sigma^{<} G^{R}+\frac{1}{2} G^{A} \Sigma^{<} G^{A},
$$

the time integration runs into future,

$$
\begin{aligned}
2 \Xi^{<}\left(t_{1}, t_{2}\right) & =\int_{-\infty}^{t_{1}} d t^{\prime} \int_{t_{2}}^{\infty} d \hat{t} G^{R}\left(t_{1}, t^{\prime}\right) \Sigma^{<}\left(t^{\prime}, \hat{t}\right) G^{R}\left(\hat{t}, t_{2}\right) \\
& +\int_{-\infty}^{t_{2}} d \hat{t} \int_{t_{1}}^{\infty} d t^{\prime} G^{A}\left(t_{1}, t^{\prime}\right) \Sigma^{<}\left(t^{\prime}, \hat{t}\right) G^{A}\left(\hat{t}, t_{2}\right) .
\end{aligned}
$$

The same integration into future also appears in the other contribution to $G^{<}$

$$
\Lambda^{<}=\frac{1}{2}\left(G^{R}-G^{A}\right) \Sigma^{<} G^{A}-\frac{1}{2} G^{R} \Sigma^{<}\left(G^{R}-G^{A}\right) .
$$

On the time diagonal, $t_{1,2}=t$, the time integrations over $t^{\prime}$ and $\hat{t}$ in $\Xi^{<}$do not overlap, see (20). The time scale of the integration is determined by the time scale of $\Sigma^{<}$that can be identified with the quasiparticle formation time 30 Thus $\Xi^{<}$is dominated by the short-time (off-pole) contributions.

The time integration in $\Lambda^{<}$extends into the future in a way that reminds Fourier transformation to energy of the asymptotic state,

$$
G^{R}\left(t, t^{\prime} ; k\right)-G^{A}\left(t, t^{\prime} ; k\right) \approx-i \mathrm{e}^{-i \varepsilon_{k}\left(t-t^{\prime}\right)} \mathrm{e}^{-\frac{\left|t-t^{\prime}\right|}{\tau}} .
$$

Thus $\Lambda^{<}$is dominated by the long-time (pole) contributions.

\section{E. Pole and off-pole contributions}

Splitting the correlation function $G^{<}$into $\Lambda^{<}$and $\Xi^{<}$ is an ideal starting point to a non-equilibrium modification of the expansion in small scattering rates. This can be seen in equilibrium, where one can use the energy representation

$$
\begin{aligned}
\lambda^{<}(\omega, k) & =f_{F D}(\omega) \frac{1}{2} a(\omega, k)^{2} \gamma(\omega) \\
& \rightarrow f(k) z(k) 2 \pi \delta(\omega-\varepsilon) .
\end{aligned}
$$

We have used $\sigma^{<}(\omega)=f_{F D}(\omega) \gamma(\omega)$, and (15). The arrow shows a value in the limit of small scattering rates.

Note that $\lambda^{<}=f_{F D} \frac{1}{2} a^{2} \gamma$, while $g^{<}=f_{F D} a$. In the limit of small scattering rate, $\gamma \rightarrow 0$, the spectral function $a$ approaches $\delta$ function as Lorentzian, while $s=\frac{1}{2} a^{2} \gamma$ approaches $\delta$ function faster,

$$
\begin{aligned}
& a(\omega, k)=\frac{\gamma}{(\omega-\epsilon-\sigma)^{2}+\frac{1}{4} \gamma^{2}}, \\
& s(\omega, k)=\frac{\frac{1}{2} \gamma^{3}}{\left((\omega-\epsilon-\sigma)^{2}+\frac{1}{4} \gamma^{2}\right)^{2}} .
\end{aligned}
$$

In the off-pole region $|\omega-\varepsilon| \gg \gamma$, the spectral function $a$ has a tail linear in $\gamma$. In the limit of small scattering rates, $\gamma \rightarrow 0$, this tail results in the off-pole correction, the second term in (16). The function $s$ in the off-pole region is proportional to $\gamma^{3}$, thus its limit is a pure $\delta$ function without the off-pole term. 
The function $\xi^{<}$contains the off-pole part. In equilibrium,

$$
\begin{aligned}
\xi^{<}(\omega, k) & =f_{F D}(\omega) \gamma(\omega) \operatorname{Re}\left(\omega-\epsilon-\sigma^{R}\right)^{2} \\
& \rightarrow f_{F D}(\omega) \gamma(\omega) \operatorname{Re}(\omega-\epsilon+i 0)^{2} .
\end{aligned}
$$

Due to the off-pole nature of this contribution, the FermiDirac distribution in (26) cannot be associated with occupation of the state $k$.

The comparison with equilibrium shows that the quasiparticle distribution relates to $\lambda^{<}$while $\xi^{<}$has to be constructed indirectly. In the spirit of the $\mathrm{BE}$, we will treat $\lambda^{<}$within a differential transport equation while $\xi^{<}$will be turned into a local functional.

Equilibrium relations (23 26) can be easily generalized to quasiclassical limit. As we have shown, the spectral function $a$ is free of gradients, see (13) and (15). The function $S=A+\frac{1}{2} G^{R} \Gamma G^{R}+\frac{1}{2} G^{A} \Gamma G^{A}$ is also free of gradients since gradient expansion of symmetric terms like $G^{R} \Gamma G^{R}$ has no gradients. Therefore, similarly to the spectral function $a$, the function $s$ just follows energy bottom defined by the field $\phi$

$$
s=\frac{\frac{1}{2} \gamma^{3}}{\left((\omega-\epsilon-\phi-\sigma)^{2}+\frac{1}{4} \gamma^{2}\right)^{2}} .
$$

In the limit of small scattering rate, $\gamma \rightarrow 0$, the function $s$ reduces to the first term of the spectral function,

$$
s=2 \pi z \delta(\omega-\varepsilon) .
$$

In equilibrium, the pole part $\lambda^{<}$is proportional to the function $s$. Out of equilibrium, we can expect similar behaviour and introduce local distribution as

$$
\lambda^{<}(\omega, k, r, t)=f_{F D}^{\text {loc }}(\omega, k, r, t) s(\omega, k, r, t) .
$$

In the limit of small scattering rates, the function $s$ turns to the $\delta$ function and one can eliminate the energy argument of the local distribution. In this way we can define the quasiparticle distribution as the pole of the local distribution. Briefly, in the limit of small scattering rates the pole part of the correlation function reads

$$
\lambda^{<}(\omega, k, r, t)=f(k, r, t) 2 \pi z \delta(\omega-\varepsilon) .
$$

The non-equilibrium generalization of the off-pole part $\xi^{<}$follows directly from its definition (19). Since symmetric terms has no gradient contributions

$$
\xi^{<}=\sigma^{<} \frac{1}{2}\left(g_{R}^{2}+g_{A}^{2}\right) .
$$

In the limit of small scattering rates

$$
\xi^{<}=\sigma^{<} \operatorname{Re} \frac{1}{(\omega-\epsilon-\phi-\sigma+i 0)^{2}} .
$$

\section{F. Boltzmann equation}

The BE is recovered from (21). First, we turn (21) into differential form multiplying it by $G_{R}^{-1}$ from the l.h.s. and by $G_{A}^{-1}$ from the r.h.s. and subtracting the two forms

$$
\begin{aligned}
- & i\left(G_{R}^{-1} \Lambda^{<}-\Lambda^{<} G_{A}^{-1}\right) \\
& =\frac{1}{2}\left(\Sigma^{<} A+A \Sigma^{<}-\Gamma G^{A} \Sigma^{<} G^{A}-G^{R} \Sigma^{<} G^{R} \Gamma\right) .
\end{aligned}
$$

By the gradient expansion, this equation simplifies as

$$
\left[\omega-\epsilon-\phi-\sigma, \lambda^{<}\right]-\frac{1}{2}\left[\gamma, a g \sigma^{<}\right]=\sigma^{<} s-\gamma \lambda^{<},
$$

where $g=\operatorname{Re} g^{R}$. In the limit of small scattering rates, the second term on the l.h.s. vanishes. After a substitution of (30), equation (34) turns into equation (3). For details see Refs. 14.29.

To deal with the transport equation (3), we need: life time $\tau$, quasiparticle energy $\varepsilon$, wave-function renormalization $z$, and transport vertex $\sigma^{<}$. Since all these functions are related to the self-energy, further progress requires to specify the self-energy. For elastic scattering on impurities, the retarded (advanced) self-energy depends only on the retarded (advanced) propagator $G^{R}$. The transport vertex depends on both propagators, $G^{R}$ and $G^{A}$, and on the correlation function $G^{<}$. Propagators are given by (13). The correlation function has to be decomposed into two parts according (18) which in Wigner's representation reads

$$
g^{<}=\lambda^{<}+\xi^{<} .
$$

The pole part $\lambda^{<}$is linked to the quasiparticle distribution via (30), the off-pole part $\xi^{<}$is self-consistently evaluated from the transport vertex $\sigma^{<}$via (32).

\section{AVERAGED T-MATRIX APPROXIMATION}

Here we specify the self-energy. As in Paper I, we assume non-interacting electrons scattered by neutral impurities. The impurity acts on electrons by single-site potential introduced by Koster and Slater13 32. Individual scattering events are treated within the T-matrix. Formulas for the T-matrix in homogeneous systems are quite common, 33 our focus will be on gradient contributions in inhomogeneous systems.

\section{A. Retarded self-energy}

In the Koster-Slater model, an impurity at position $r$ is characterized by a potential restricted to a single orbital $|r\rangle$ at site $r$,

$$
V_{r}=|r\rangle v\langle r| .
$$


Corresponding retarded T-matrix reads

$$
T_{r}^{R}=V_{r}+V_{r} G^{R} T_{r}^{R} .
$$

Iterating (37) one can see that the T-matrix is also singlesite function,

$$
T_{r}^{R}=|r\rangle \frac{v}{1-\left\langle r\left|G^{R}\right| r\right\rangle}\langle r| .
$$

The T-matrix does not depend on difference coordinate, therefore its mixed representation relates only to double-time structure

$$
|r\rangle t^{R}(\omega, t, r)\left\langle r\left|=\int d \tau \mathrm{e}^{i \omega \tau}\right| r\right\rangle t^{R}\left(t+\frac{\tau}{2}, t-\frac{\tau}{2}, r\right)\langle r| .
$$

Similarly, the local element of the propagator also depends only on energy, not on momentum. In Wigner's representation, the local element of the retarded propagator $\left\langle r\left|G^{R}\left(t_{1}, t_{2}\right)\right| r\right\rangle \equiv G^{R}\left(r, t_{1}, r, t_{2}\right) \equiv \tilde{G}^{R}\left(r, t_{1}, t_{2}\right)$ transforms into

$$
\tilde{g}^{R}(\omega, r, t)=\int \frac{d k}{(2 \pi)^{3}} g^{R}(\omega, k, r, t) .
$$

The T-matrix in mixed representation reads

$$
t^{R}(\omega, r, t)=\frac{v}{1-v \tilde{g}^{R}(\omega, r, t)} .
$$

There are no gradient contributions. This can be checked directly by explicit gradient expansion of (38).

The retarded self-energy is defined as a mean value of the T-matrix

$$
\Sigma^{R}=\int d r c(r) T_{r}^{R}
$$

where $c(r)$ is a concentration of impurity per site on site $r$. This approximation is called the self-consistent averaged T-matrix approximation (ATA). Unlike in Paper I, we do not use the subscript self here since the self-consistent form is a natural starting point in transport theory. Non-self-consistent values are introduced and denoted below.

In Wigner's representation (42) reads

$$
\sigma^{R}(\omega, r, t)=c(r) t^{R}(\omega, r, t) .
$$

\section{B. Transport vertex}

The transport vertex $\sigma^{<}$in the self-consistent ATA depends on the correlation function $G^{<}$as

$$
\Sigma^{<}=\int d r c(r) T_{r}^{R} G^{<} T_{r}^{A},
$$

which in Wigner's representation reads

$$
\begin{aligned}
\sigma^{<} & =c t^{R} \tilde{g}^{<} t^{A} \\
& +c \frac{i}{2} \tilde{g}^{<}\left[t^{R}, t^{A}\right] \\
& +c \frac{i}{2}\left(t^{A}\left[t^{R}, \tilde{g}^{<}\right]-t^{R}\left[t^{A}, \tilde{g}^{<}\right]\right) .
\end{aligned}
$$

Here,

$$
\tilde{g}^{<}(\omega, r, t)=\int \frac{d k}{(2 \pi)^{3}} G^{<}(\omega, k, r, t)
$$

is the local element of the correlation function. The Poisson bracket used in (45) as a short-hand for gradient corrections in general includes space derivatives combined with derivatives with respect to momentum, see (11). In (45), however, none of functions depends on momentum so that the only gradient contributions come from timederivatives.

The transport vertex $\sigma^{<}$has three basic components that have distinguishable physical content. First, there is a non-gradient term

$$
\sigma_{\mathrm{ng}}^{<}=c t^{R} \tilde{g}^{<} t^{A}
$$

Second, there is the term

$$
\sigma_{\mathrm{P}}^{<}=c \frac{i}{2} \tilde{g}^{<}\left[t^{R}, t^{A}\right]
$$

which formally brings gradient corrections to the scattering rates. Below we show that this term vanishes. Third, there are two complex conjugate terms

$$
\sigma_{\Delta}^{<}=c \frac{i}{2}\left(t^{A}\left[t^{R}, \tilde{g}^{<}\right]-t^{R}\left[t^{A}, \tilde{g}^{<}\right]\right)
$$

which contribute if the quasiparticle distribution $f$ has non-zero gradients. This last term results in non-local corrections of the BE (11).

Now the set of equations is complete.

\section{SCATTERING INTEGRALS}

The scattering-out integral $z \gamma_{\varepsilon} f$ and the non-gradient part of the scattering-in integral $z \sigma_{\varepsilon}^{<}$are dominant. They are the only non-zero terms of the BE in the absence of the perturbing field $\phi$ and their balance determines how the $\mathrm{BE}$ is capable to describe equilibrium.

\section{A. Non-gradient part of the scattering integral}

In the quasiclassical limit, the frequency of the perturbing field is much smaller than the relaxation time of the system, i.e., all perturbations are on the long-time scale. Therefore, the consistency of $z \gamma_{\varepsilon} f$ and $z \sigma_{\varepsilon}^{<}$is crucial and it has to be checked in very detail. 
On the level of non-equilibrium Green's functions, the scattering in and out are consistent if the self-energy is given by the T-matrix that obeys the optical theorem. The ATA obeys it. The consistency on the level of Green's functions does not imply a consistency within the BE. The scattering-in integral in the BE include additional approximations (30) and (32), while the scattering out is evaluated without them. Since (30) and (32) follow from the limit of small scattering rates, we have to make corresponding approximation for the scattering out.

\section{Scattering out}

The limit of small scattering rates, $\gamma \rightarrow 0$, is conveniently discussed in terms of the non-self-consistent ATA. Indeed, sending $\sigma^{R} \rightarrow 0$ in propagators $\tilde{g}^{R}$ of the selfconsistent T-matrix $t^{R}$ turns into the non-self-consistent one $t_{00}^{R}$.

¿From (12) one can see that

$$
g^{R}(\omega)=g_{00}^{R}\left(\omega-\phi-\sigma^{R}\right)
$$

where subscript 00 denotes no field and no self-energy in propagator, [in Paper I, these non-self-consistent functions are without subscript]

$$
g_{00}^{R}(\omega)=\frac{1}{\omega-\epsilon+i 0} .
$$

The self-energy $\sigma^{R}$ is then expressed in terms of the nonself-consistent self-energy

$$
\sigma_{00}^{R}=c t_{00}^{R}=c \frac{v}{1-v \tilde{g}_{00}^{R}}
$$

as

$$
\sigma^{R}(\omega)=\sigma_{00}^{R}\left(\omega-\phi-\sigma+\frac{i}{2} \gamma\right)
$$

In the limit of small scattering rates we linearize in $\gamma$,

$$
\sigma^{R}(\omega)=\sigma_{00}^{R}(\omega-\phi-\sigma)+\left.\frac{i}{2} \gamma \frac{\partial \sigma_{00}^{R}}{\partial \omega}\right|_{\omega-\phi-\sigma}
$$

The imaginary part of (54), $\gamma \equiv-2 \operatorname{Im} \sigma^{R}$, reads

$$
\gamma(\omega)\left(1+\frac{\partial \sigma_{00}}{\partial \omega}\right)_{\omega-\phi-\sigma}=\gamma_{00}(\omega-\phi-\sigma) .
$$

At the pole value $\omega=\varepsilon=\epsilon+\phi+\sigma(\varepsilon)$, the argument of $\gamma_{00}$ simplifies

$$
\gamma(\varepsilon)\left(1+\frac{\partial \sigma_{00}}{\partial \omega}\right)_{\omega=\epsilon}=\gamma_{00}(\epsilon)
$$

The factor $1+\left.\frac{\partial \sigma_{00}}{\partial \omega}\right|_{\omega=\epsilon}$ is just the wave-function renormalization $z$, see Appendix A of Paper I. Indeed, from real part of (54) one finds

$$
\left.\frac{\partial \sigma}{\partial \omega}\right|_{\omega=\varepsilon}=\left.\frac{\partial \sigma_{00}}{\partial \omega}\right|_{\omega=\varepsilon-\phi-\sigma}\left(1-\left.\frac{\partial \sigma}{\partial \omega}\right|_{\omega=\varepsilon}\right),
$$

which is equivalent to

$$
z=\frac{1}{1-\left.\frac{\partial \sigma}{\partial \omega}\right|_{\omega=\varepsilon}}=1+\left.\frac{\partial \sigma_{00}}{\partial \omega}\right|_{\omega=\epsilon} .
$$

Accordingly, we have found that the self-consistent and non-self-consistent approximations are linked via the wave-function renormalization

$$
\frac{1}{\tau}=z \gamma_{\varepsilon}=\gamma_{00}(\epsilon)=c(-2) \operatorname{Im} t_{00}^{R}(\epsilon) .
$$

The non-self-consistent T-matrix satisfies the optical theorem $\operatorname{Im} t_{00}^{R}=t_{00}^{R}\left(\operatorname{Im} \tilde{g}_{00}^{R}\right) t_{00}^{A}$, which easily follows from (41) and the complex conjugacy of retarded and advanced functions, $t_{00}^{A}=\left(t_{00}^{R}\right)^{*}$. The scattering-out rate thus can be expressed in form of a sum over individual scattering rates into all accessible finite states

$$
z \gamma_{\varepsilon_{k}}=c\left|t_{00}^{R}\left(\epsilon_{k}\right)\right|^{2} \int \frac{d p}{(2 \pi)^{3}} 2 \pi \delta\left(\epsilon_{k}-\epsilon_{p}\right) .
$$

The $\delta$ function in the r.h.s. results from $-2 \operatorname{Im} g_{00}^{R}(\omega)=$ $2 \pi \delta(\omega-\epsilon)$. From (60) one identifies the scattering rates

$$
P_{p k}=c\left|t_{00}^{R}\left(\epsilon_{k}\right)\right|^{2} 2 \pi \delta\left(\epsilon_{k}-\epsilon_{p}\right)
$$

Since there are no gradient contributions to the scattering out, the quantum-mechanical scattering-out rate (61) is of the same form as the intuitively expected classical scattering-out integral in the modified BE (1).

\section{Scattering in}

Now we evaluate the non-gradient part $\sigma_{\text {ng }}^{<}$of the scattering-in integral (47). We show that it results exactly in the scattering-in integral from the BE (11) with the scattering rates given by (61). In this way, the consistency of scattering in and out will be checked.

Substituting $\tilde{g}^{<}$from (46) with $g^{<}=\lambda^{<}+\xi^{<}$from (30) and (32), one gets

$$
\begin{aligned}
\sigma_{\mathrm{ng}}^{<} & =c\left|t^{R}\right|^{2} \tilde{g}^{<} \\
& =c\left|t^{R}\right|^{2} \int \frac{d p}{(2 \pi)^{3}} z f(p) 2 \pi \delta\left(\omega-\varepsilon_{p}\right) \\
& +c\left|t^{R}\right|^{2} \int \frac{d p}{(2 \pi)^{3}} \sigma_{\mathrm{ng}}^{<} \operatorname{Re} \frac{1}{\left(\omega-\epsilon_{p}-\phi-\sigma+i 0\right)^{2}} .
\end{aligned}
$$

The first term follows from $\lambda^{<}$and is the dominant one. The second one is the off-pole correction due to $\xi^{<}$.

The T-matrix in 62 includes propagators with poles out of the real energy axis, shifted by $\gamma / 2$. In the limit of small scattering rates, it is advantageous to use the non-self-consistent ATA as the reference point 


$$
\begin{aligned}
t^{R}(\omega) & =t_{00}^{R}\left(\omega-\phi-\sigma+\frac{i}{2} \gamma\right) \\
& =t_{00}^{R}(\omega-\phi-\sigma)+\left.\frac{i}{2} \gamma \frac{\partial t_{00}^{R}}{\partial \omega}\right|_{\omega-\phi-\sigma} .
\end{aligned}
$$

¿From this approximation one finds that the square of the absolute value at the pole reads

$$
\begin{aligned}
\left|t^{R}(\varepsilon)\right|^{2} & =\left|t_{00}^{R}(\epsilon)\right|^{2} \\
& \times\left(1+\gamma_{\varepsilon} \frac{i}{2}\left(\frac{1}{t_{00}^{R}} \frac{\partial t_{00}^{R}}{\partial \omega}-\frac{1}{t_{00}^{A}} \frac{\partial t_{00}^{A}}{\partial \omega}\right)_{\omega=\epsilon}\right) .
\end{aligned}
$$

The second term in the bracket in (64) can be expressed in terms of the collision delay 34 (I-25)

$$
\Delta_{t}=\left.\operatorname{Im} \frac{1}{t_{00}^{R}} \frac{\partial t_{00}^{R}}{\partial \omega}\right|_{\omega=\epsilon}=\frac{i}{2}\left(\frac{1}{t_{00}^{R}} \frac{\partial t_{00}^{R}}{\partial \omega}-\frac{1}{t_{00}^{A}} \frac{\partial t_{00}^{A}}{\partial \omega}\right)_{\omega=\epsilon}
$$

as

$$
\left|t^{R}(\varepsilon)\right|^{2}=\left|t_{00}^{R}(\epsilon)\right|^{2}\left(1-\frac{\Delta_{t}}{\tau} \frac{1}{z}\right) .
$$

This relation between the self-consistent and non-selfconsistent T-matrices has a form of virial corrections. These are not, however, the virial corrections we are looking for. The term $\frac{\Delta_{t}}{\tau}$ will be cancelled by the wavefunction renormalization and the off-pole contribution.

Let us denote by $\sigma_{00}^{<}$the non-self-consistent-like scattering vertex

$$
\begin{aligned}
\sigma_{00}^{<}\left(\varepsilon_{k}\right) & =c\left|t_{00}^{R}\left(\varepsilon_{k}-\phi-\sigma\right)\right|^{2} \int \frac{d p}{(2 \pi)^{3}} z f(p) 2 \pi \delta\left(\varepsilon_{k}-\varepsilon_{p}\right) \\
& =c\left|t_{00}^{R}\left(\epsilon_{k}\right)\right|^{2} \int \frac{d p}{(2 \pi)^{3}} f(p) 2 \pi \delta\left(\epsilon_{k}-\epsilon_{p}\right) \\
& =\int \frac{d p}{(2 \pi)^{3}} P_{p k} f(p) .
\end{aligned}
$$

Apparently, $\sigma_{00}^{<}$is compatible with the scattering out. To prove the compatibility of $\sigma_{\text {ng }}^{<}$with $f \gamma$, we have to show that all corrections (linear in $\gamma$ ) following from the non-self-consistency mutually cancel, i.e., $z \sigma_{\mathrm{ng}}^{<}=\sigma_{00}^{<}$.

There are three contributions to the correction $z \sigma_{\text {ng }}^{<}-$ $\sigma_{00}^{<}$: (i) the second term in (62), (ii) the $\frac{\Delta_{t}}{\tau}$ correction to the square of the T-matrix, and (iii) the wave-function renormalization in front of $\sigma_{\varepsilon}^{<}$. Remains to show that the sum of these three corrections vanishes. We will neglect higher order terms resulting from products of individual corrections.

First, we rearrange the second term in (62). To this end we use that $\sigma^{<}$does not depend on the momentum $p$ and move it out of the integral. The integrand can be rearranged is spirit of Ward's identities with help of energy derivative as

$$
\operatorname{Re} \frac{1}{(\omega-\epsilon-\phi-\sigma+i 0)^{2}}=-\left.\frac{\partial g_{00}}{\partial \omega} \frac{1}{1-\frac{\partial \sigma}{\partial \omega}}\right|_{\omega-\phi-\sigma} .
$$

By the integration over $p$, relation (68) turns into the local propagator needed in the second term of (62)

$$
\int \frac{d p}{(2 \pi)^{3}} \operatorname{Re} \frac{1}{\left(\varepsilon_{k}-\epsilon_{p}-\phi-\sigma\left(\varepsilon_{k}\right)+i 0\right)^{2}}=-\left.z \frac{\partial \tilde{g}_{00}}{\partial \omega}\right|_{\epsilon_{k}} .
$$

The second term of (62) thus can be rearranged as

$$
\begin{aligned}
& c\left|t^{R}\right|^{2} \int \frac{d p}{(2 \pi)^{3}} \sigma_{\mathrm{ng}}^{<} \operatorname{Re} \frac{1}{\left(\varepsilon_{k}-\epsilon_{p}-\phi-\sigma\left(\varepsilon_{k}\right)+i 0\right)^{2}} \\
& =-\left.z \sigma_{\mathrm{ng}}^{<} c\left|t_{00}^{R}\left(\epsilon_{k}\right)\right|^{2}\left(1-\frac{\Delta_{t}}{\tau} \frac{1}{z}\right) \frac{\partial \tilde{g}_{00}}{\partial \omega}\right|_{\epsilon_{k}} .
\end{aligned}
$$

Now we can collect all terms which contribute to the non-gradient part of the scattering in

$$
\begin{aligned}
z \sigma_{\mathrm{ng}}^{<}\left(\varepsilon_{k}\right) & =\left(z-\frac{\Delta_{t}}{\tau}\right) \sigma_{00}^{<}\left(\varepsilon_{k}\right)-\left.c\left|t_{00}^{R}\right|^{2} \frac{\partial \tilde{g}_{00}}{\partial \omega}\right|_{\epsilon_{k}} z \sigma_{\mathrm{ng}}^{<}\left(\varepsilon_{k}\right) \\
& =\left(1+\frac{\partial \sigma_{00}}{\partial \omega}-\frac{\Delta_{t}}{\tau}-c\left|t_{00}^{R}\right|^{2} \frac{\partial \tilde{g}_{00}}{\partial \omega}\right)_{\epsilon_{k}} \sigma_{00}^{<}\left(\varepsilon_{k}\right) \\
& =\sigma_{00}^{<}\left(\varepsilon_{k}\right) .
\end{aligned}
$$

In the last but one step, we have neglected the crosscorrection $\frac{\Delta_{t}}{\tau} \times c\left|t_{00}^{R}\right|^{2} \frac{\partial \tilde{g}_{00}}{\partial \omega}$ and terms quadratic in $c\left|t_{00}^{R}\right|^{2} \frac{\partial \tilde{g}_{00}}{\partial \omega}$ which are higher order in the limit of small scattering rates. In the last step, we have used the derived optical theorem (I-B3), [proved also in Appendix of this paper, see (A17)]

$$
\frac{\Delta_{t}}{\tau}=\left(\frac{\partial \sigma_{00}}{\partial \omega}-c\left|t_{00}^{R}\right|^{2} \frac{\partial \tilde{g}_{00}}{\partial \omega}\right)_{\epsilon_{k}} .
$$

Briefly, we have shown that in the non-gradient scattering in and out, the wave-function renormalization $z$ compensates in a consistent manner. This compensation shows that the off-pole part $\Xi^{<}$of the correlation function is capable to rebound consistently the off-pole portion of the particle propagation.

The non-gradient parts of scattering integrals

$$
\begin{aligned}
z \gamma\left(\varepsilon_{k}, r, t\right) & =\int \frac{d k}{(2 \pi)^{3}} P_{k p} f(k, r, t), \\
z \sigma_{\mathrm{ng}}^{<}\left(\varepsilon_{k}, r, t\right) & =\int \frac{d k}{(2 \pi)^{3}} P_{p k} f(p, r, t),
\end{aligned}
$$

have scattering rates identical to their non-self-consistent counterparts

$$
\begin{aligned}
P_{p k} & =c\left|t_{00}^{R}\left(\epsilon_{k}\right)\right|^{2} 2 \pi \delta\left(\epsilon_{k}-\epsilon_{p}\right) \\
& =\frac{1}{\tau} \frac{2 \pi^{2}}{k^{2}} \delta(|p|-|k|) .
\end{aligned}
$$

These non-gradient parts are identical to those used in the intuitive BE (2). 


\section{B. Gradient corrections to the scattering rate}

The gradient term of the self-energy $\sigma_{\mathrm{P}}^{<}$given by 48 does not include gradients of the quasiparticle distribution. Accordingly, $\sigma_{\mathrm{P}}^{<}$can bring corrections to scattering rates $P_{p k}$ only. In reality $\sigma_{\mathrm{P}}^{<}=0$, i.e., no correction to scattering rates appears.

For impurity potentials independent from time, $v=$ const, the Poisson bracket in (48) equals zero. Indeed, the both T-matrices depend on the time $t$ only via the internal potential $\phi$ which always enters the T-matrix in the form of $\omega-\phi$, i.e., $t^{R, A}(\omega, t)=t^{R, A}(\omega-\phi)$. Any two functions of this property has zero Poisson bracket,

$$
\begin{aligned}
{[a(\omega-\phi), b(\omega-\phi)] } & =\frac{\partial a}{\partial \omega} \frac{\partial b}{\partial t}-\frac{\partial a}{\partial t} \frac{\partial b}{\partial \omega} \\
& =-\frac{\partial a}{\partial \omega} \frac{\partial b}{\partial \omega} \frac{\partial \phi}{\partial t}+\frac{\partial a}{\partial \omega} \frac{\partial \phi}{\partial t} \frac{\partial b}{\partial \omega} \\
& =0 .
\end{aligned}
$$

Accordingly,

$$
z \sigma_{\mathrm{P}}^{<}=0
$$

Briefly, amplitudes of scattering rates are not modified by gradient corrections for time-independent impurity potentials. This is in agreement with the intuitive expectation used in Paper I.

\section{Gradient corrections to scattering in}

The gradient correction $\sigma_{\Delta}^{<}$brings true non-local contributions to the scattering-in integral. Via gradients of the local correlation function $\tilde{g}^{<}$, the correction $\sigma_{\Delta}^{<}$depends on gradients of the quasiparticle distribution. Here we show that from $\sigma_{\Delta}^{<}$one recovers virial corrections in form discussed in Paper I.

First, we write (49) in explicit form

$$
\begin{aligned}
\sigma_{\Delta}^{<} & =c t^{R} t^{A} \frac{i}{2}\left(\frac{1}{t^{R}}\left[t^{R}, \tilde{g}^{<}\right]-\frac{1}{t^{A}}\left[t^{A}, \tilde{g}^{<}\right]\right) \\
& =-c\left|t^{R}\right|^{2} \operatorname{Im} \frac{1}{t^{R}}\left[t^{R}, \tilde{g}^{<}\right] \\
& =-c\left|t^{R}\right|^{2} \operatorname{Im} \frac{1}{t^{R}}\left(\frac{\partial t^{R}}{\partial \omega} \frac{\partial}{\partial t}-\frac{\partial t^{R}}{\partial t} \frac{\partial}{\partial \omega^{<}}\right) \tilde{g}^{<} .
\end{aligned}
$$

Now, we substitute the dominant part of the local correlation function (the off-pole part leads to higher order contribution in small $\gamma$ ) into 49 )

$$
g^{<}(\omega, r, t) \approx \int \frac{d p}{(2 \pi)^{3}} f(p, r, t) 2 \pi z \delta(\omega-\varepsilon)
$$

and interchange derivatives with the momentum integral

$$
\begin{aligned}
\sigma_{\Delta}^{<} & =-c\left|t^{R}\right|^{2} \int \frac{d p}{(2 \pi)^{3}} \operatorname{Im} \frac{1}{t^{R}} \\
& \times\left(\frac{\partial t^{R}}{\partial \omega} \frac{\partial}{\partial t}-\frac{\partial t^{R}}{\partial t} \frac{\partial}{\partial \omega}\right) f(p) 2 \pi z \delta\left(\omega-\varepsilon_{p}\right) .
\end{aligned}
$$

The function $z \delta(\omega-\varepsilon)$ depends on time only via $\omega-\phi$ $[z \delta(\omega-\varepsilon)=\delta(\omega-\epsilon-\phi-\sigma)]$, therefore according to (76), $\left(\frac{\partial t^{R}}{\partial \omega} \frac{\partial}{\partial t}-\frac{\partial t^{R}}{\partial t} \frac{\partial}{\partial \omega}\right) 2 \pi z \delta\left(\omega-\varepsilon_{p}\right)=0$. The gradient correction $\sigma_{\Delta}^{<}$thus depends exclusively on gradients of the distribution function,

$$
\sigma_{\Delta}^{<}=-c\left|t^{R}\right|^{2} \int \frac{d p}{(2 \pi)^{3}} 2 \pi z \delta\left(\omega-\varepsilon_{p}\right) \operatorname{Im} \frac{1}{t^{R}} \frac{\partial t^{R}}{\partial \omega} \frac{\partial}{\partial t} f(p) .
$$

Finally, we simplify this gradient correction with help of (66), scattering rate (61) and the collision delay (65) as

$$
\begin{aligned}
z \sigma_{\Delta}^{<} & =-\left(z-\frac{\Delta_{t}}{\tau}\right) c\left|t_{00}^{R}\right|^{2} \int \frac{d p}{(2 \pi)^{3}} 2 \pi z \delta(\omega-\varepsilon) \Delta_{t} \frac{\partial}{\partial t} f(p) \\
& =-\int \frac{d p}{(2 \pi)^{3}} P_{p k} \frac{\partial f}{\partial t} \Delta_{t}
\end{aligned}
$$

In the second line, we have neglected higher order terms using the approximation $z-\Delta_{t} / \tau \approx 1$.

This gradient correction to the scattering integrals has the form of virial corrections, the last term of (2). In particular, no other gradient than the time derivative of the quasiparticle distribution appears, and this time derivative is weighted with the collision delay and the scattering rate.

\section{RECOVERING THE INTUITIVE TRANSPORT EQUATION}

Now we can put together elements of the transport equation (3) and reconstruct (11).

\section{A. Drift part of the transport equation}

The velocity results from momentum derivative of (14) as

$$
\begin{aligned}
\frac{\partial \varepsilon}{\partial k} & =\frac{\partial \epsilon}{\partial k}+\left.\frac{\partial \sigma(\omega)}{\partial \omega}\right|_{\omega=\varepsilon} \frac{\partial \varepsilon}{\partial k} \\
& =\frac{1}{1-\left.\frac{\partial \sigma(\omega)}{\partial \omega}\right|_{\omega=\varepsilon}} \frac{\partial \epsilon}{\partial k} .
\end{aligned}
$$

For parabolic kinetic energy, $\epsilon=\frac{k^{2}}{2 m}$, the quasiparticle velocity gains the form used in the $\mathrm{BE}$ (11),

$$
\frac{\partial \varepsilon}{\partial k}=z \frac{k}{m}
$$

The force acting on quasiparticle is also found from the quasiparticle energy,

$$
\frac{\partial \varepsilon}{\partial r}=\frac{\partial \phi}{\partial r}+\frac{\partial \sigma}{\partial r}+\frac{\partial \sigma}{\partial \omega} \frac{\partial \varepsilon}{\partial r}
$$


For homogeneous distribution of impurities, the selfenergy depends on coordinate $r$ only via potential $\phi$. Since the potential $\phi$ can be viewed as a local shift of initial of energies, one finds that the self-energy $\sigma$ relates to the self-energy $\sigma_{\phi=0}$ in the absence of the field $\phi$ as

$$
\sigma(\omega)=\sigma_{\phi=0}(\omega-\phi)
$$

¿From (86) one finds that the second term of the force (85) reads

$$
\frac{\partial \sigma}{\partial r}=-\frac{\partial \sigma}{\partial \omega} \frac{\partial \phi}{\partial r}
$$

Using (87) in (85), one finds that the force has no renormalization

$$
\frac{\partial \varepsilon}{\partial r}=\frac{\partial \phi}{\partial r}
$$

which is the form of the force in the BE (11). Thus drift terms of (3) reduce to drift terms of (11).

\section{B. Scattering integral with virial corrections}

To obtain the expected form (11), we use that within linear approximation

$$
a(x)+\frac{\partial a}{\partial x} \Delta=a(x+\Delta) .
$$

Thus the non-gradient and the gradient scattering-in contributions can be collected into a compact expression

$$
\begin{aligned}
z \sigma_{\varepsilon}^{<} & =z \sigma_{\mathrm{ng}}^{<}\left(\varepsilon_{k}\right)+z \sigma_{\Delta}^{<}\left(\varepsilon_{k}\right) \\
& =\int \frac{d p}{(2 \pi)^{3}} P_{p k}\left(f-\frac{\partial f}{\partial t} \Delta_{t}\right) \\
& =\int \frac{d p}{(2 \pi)^{3}} P_{p k} f\left(p, r, t-\Delta_{t}\right) \\
& =\frac{1}{\tau} \frac{2 \pi^{2}}{k^{2}} \int \frac{d p}{(2 \pi)^{3}} \delta(|p|-|k|) f\left(p, r, t-\Delta_{t}\right) .
\end{aligned}
$$

One can see that the scattering in has the form expected from classical assumptions.

Substituting (59) for the scattering out, (90) for the scattering in, 84) for the quasiparticle velocity, and (88) for the accelerating force into the asymptotic equation (3), the intuitive modification of the BE (11) is recovered from quantum statistics.

\section{OBSERVABLES}

To recover the transport equation (11) was our major task. With respect to applications, one has also to find relation between quasiparticle distribution $f$ and observables. As already shown in Paper I, these relations include quasiparticle and virial corrections. In Paper I we have discussed only those observables that can be identified from the transport equation via conservation laws. Here we extend our treatment to a general single-particle observable.

All single-electron observables can be expressed in terms of the reduced density matrix (Wigner's distribution function)

$$
\rho(k, r, t)=\int \frac{d \omega}{2 \pi} G^{<}(\omega, k, r, t)
$$

¿From the decomposition $g^{<}=\lambda^{<}+\xi^{<}$, where $\lambda^{<}$and $\xi<$ are given by (30) and (32), respectively, one finds the reduced density matrix as

$$
\rho=z f-\int \frac{d \omega}{2 \pi} \frac{\wp}{\omega-\varepsilon} \frac{\partial \sigma^{<}}{\partial \omega} .
$$

Note that the transport vertex $\sigma^{<}$in (92) does not enters the reduced density only by its pole value $\sigma_{\varepsilon}^{<}$but the full energy dependence has to be maintained. Since the second term is already an off-pole correction, the correlation function of the self-energy is used only in its lowest approximation $\sigma^{<}=\sigma_{00}^{<}$.

The formula (92) has no explicit gradient terms, however, there are gradient contributions hidden in the transport vertex $\sigma^{<}$. There is a question whether one should keep these gradient corrections in the off-pole part of formula (92) or not. A general answer is not clear to us. On the other hand, with respect to conservation laws that test a consistency of observables with the transport equation (11), the gradient contributions can be neglected. The transport equation does not provide observables but only their time or space derivatives, see e.g. the equation of continuity (I-C2), therefore any gradient contribution to observables enter the conservation law via second derivatives that are neglected within the quasiclassical limit. Accordingly, we neglect gradient corrections to $\sigma^{<}$ in (92).

\section{A. Local density of electrons}

In Paper I we have derived the local density of electrons $n$ from the transport equation, see (I-5). For scattering on the Koster-Slater impurities it was found that the correlated density $n_{\text {corr }}$ is determined by the ratio of the collision delay to the lifetime, see (I-29). Here we recover (I-29) directly from (92).

The local density of quasiparticles reads

$$
n_{\text {free }}=\int \frac{d k}{(2 \pi)^{3}} f(k) \text {. }
$$

The local density of electrons is given by the integral from $\rho$ over momentum

$$
n=\int \frac{d k}{(2 \pi)^{3}} \rho .
$$


¿From (94) and (92) one finds

$$
\begin{aligned}
n & =\int \frac{d k}{(2 \pi)^{3}} f(k) \\
& +\left.\int \frac{d k}{(2 \pi)^{3}} \frac{\partial \sigma_{00}}{\partial \omega}\right|_{\omega=\epsilon_{k}} f(k) \\
& +\int \frac{d k}{(2 \pi)^{3}} \int \frac{d \omega}{2 \pi} \frac{\wp}{\omega-\varepsilon_{k}} \frac{\partial \sigma_{00}^{<}(\omega)}{\partial \omega},
\end{aligned}
$$

where we have used (58) for the wave-function renormalization $z$. The first term is the quasiparticle density $n_{\text {free }}$, the second and the third terms are the correlated density

$$
\begin{aligned}
n_{\text {corr }} & =\left.\int \frac{d k}{(2 \pi)^{3}} \frac{\partial \sigma_{00}}{\partial \omega}\right|_{\omega=\epsilon_{k}} f(k) \\
& +\int \frac{d k}{(2 \pi)^{3}} \int \frac{d \omega}{2 \pi} \frac{\wp}{\omega-\varepsilon_{k}} \frac{\partial \sigma_{00}^{<}(\omega)}{\partial \omega} .
\end{aligned}
$$

In the second term we perform the integration by parts

$$
\frac{\partial}{\partial \omega} \frac{\wp}{\omega-\varepsilon}=-\operatorname{Re} \frac{1}{(\omega-\varepsilon+i 0)^{2}},
$$

and substitute for the self-energy $\sigma_{00}^{<}$from (67)

$$
\begin{aligned}
n_{\text {corr }} & =\left.\int \frac{d k}{(2 \pi)^{3}} f(k) \frac{\partial \sigma_{00}}{\partial \omega}\right|_{\omega=\varepsilon_{k}} \\
& +\int \frac{d k}{(2 \pi)^{3}} \frac{d \omega}{2 \pi} \operatorname{Re} \frac{1}{\left(\omega-\varepsilon_{k}+i 0\right)^{2}} c\left|t_{00}^{R}(\omega-\sigma-\phi)\right|^{2} \\
& \times \int \frac{d p}{(2 \pi)^{3}} z f(p) 2 \pi \delta\left(\omega-\varepsilon_{p}\right) .
\end{aligned}
$$

Due to the energy-conserving $\delta$ function, the energy $\omega$ can be easily integrated out. The wave-function renormalization $z$ under the $p$ integral can be omitted as higher order in the limit of small scattering rates,

$$
\begin{aligned}
n_{\text {corr }} & =\left.\int \frac{d k}{(2 \pi)^{3}} f(k) \frac{\partial \sigma_{00}}{\partial \omega}\right|_{\omega=\epsilon_{k}} \\
& +c \int \frac{d k}{(2 \pi)^{3}} \frac{d p}{(2 \pi)^{3}} f(p) \operatorname{Re} \frac{1}{\left(\epsilon_{p}-\epsilon_{k}+i 0\right)^{2}}\left|t_{00}^{R}\left(\epsilon_{p}\right)\right|^{2} .
\end{aligned}
$$

We have used (14) to simplify energy arguments. Now we can integrate over momentum $k$ using non-self-consistent version of (69),

$$
\begin{aligned}
n_{\text {corr }} & =\left.\int \frac{d k}{(2 \pi)^{3}} f(k) \frac{\partial \sigma_{00}}{\partial \omega}\right|_{\omega=\epsilon_{k}} \\
& -\left.c \int \frac{d p}{(2 \pi)^{3}} f(p) \frac{\partial \tilde{g}_{00}}{\partial \omega}\right|_{\omega=\epsilon_{p}}\left|t_{00}^{R}\left(\epsilon_{p}\right)\right|^{2} .
\end{aligned}
$$

The two terms can be joint. In the first term we rename the integration variable $k$ to $p$, so that both terms will have the same name of the momentum argument of the distribution function $f$,

$$
n_{\mathrm{corr}}=\int \frac{d p}{(2 \pi)^{3}} f(p)\left(\frac{\partial \sigma_{00}}{\partial \omega}-c\left|t_{00}^{R}\right|^{2} \frac{\partial \tilde{g}_{00}}{\partial \omega}\right)_{\omega=\epsilon_{p}} .
$$

Finally, we apply the derived optical theorem (72) to recover the relation (I-29)

$$
n_{\text {corr }}=\int \frac{d p}{(2 \pi)^{3}} f(p) \frac{\Delta_{t}}{\tau} .
$$

The total density $n=n_{\text {free }}+n_{\text {corr }}$ resulting from the reduced density matrix (92) is thus identical to the one obtained from the transport equation via the equation of continuity,

$$
n=\int \frac{d p}{(2 \pi)^{3}} f(p)\left(1+\frac{\Delta_{t}}{\tau}\right),
$$

which is Eq (I-48). Briefly, with respect to the electron density, the approximative functional for the reduced density matrix 92 is consistent with approximations in the transport equation (11).

\section{B. Local density of current}

The particle current is one of the quantities most often evaluated from the BE. Here we show that for the KosterSlater impurities there are no explicit virial corrections in the functional for current.

A general formula for the current is

$$
j=\int \frac{d k}{(2 \pi)^{3}} \frac{\partial \epsilon}{\partial k} \rho=\int \frac{d k}{(2 \pi)^{3}} \frac{k}{m} \rho .
$$

Now we substitute for $\rho$ from (92)

$$
\begin{aligned}
j & =\int \frac{d k}{(2 \pi)^{3}} \frac{k}{m} z f \\
& +\int \frac{d k}{(2 \pi)^{3}} \frac{d \omega}{2 \pi} \frac{k}{m} \operatorname{Re} \frac{\sigma^{<}(\omega)}{\left(\omega-\varepsilon_{k}+i 0\right)^{2}} \\
& =\int \frac{d k}{(2 \pi)^{3}} \frac{k}{m} z f .
\end{aligned}
$$

The second term in the first line is zero because its integrand is an odd function of momentum $k$. The current is thus identical to (I-41) found from the equation of continuity.

\section{Local density of energy}

Energy of the system is not a single-electron observable. Although electrons do not mutually interact, the energy of the system cannot be evaluated from the reduced density matrix $\rho$. This is because electrons are correlated with impurities of unknown positions. Similarly to interacting systems, the energy has to be evaluated directly from the correlation function $g^{<}$, 


$$
E=\int \frac{d \omega}{2 \pi} \frac{d k}{(2 \pi)^{3}} \omega g^{<}(\omega, k) .
$$

To express the energy in terms of quasiparticle distribution, we use $g^{<}=\lambda^{<}+\xi^{<}$with $\lambda^{<}$from (30) and $\xi^{<}$ from (32),

$$
\begin{aligned}
E & =\int \frac{d \omega}{2 \pi} \frac{d k}{(2 \pi)^{3}} \omega\left(\left(1-\frac{\partial \sigma}{\partial \omega}\right)^{-1} f(k) 2 \pi \delta\left(\omega-\varepsilon_{k}\right)\right. \\
& +c\left|t^{R}(\omega)\right|^{2} \int \frac{d p}{(2 \pi)^{3}} f(p) 2 \pi \delta\left(\omega-\varepsilon_{p}\right) \\
& \left.\times \operatorname{Re} \frac{1}{\left(\omega-\varepsilon_{k}+i 0\right)^{2}}\right)
\end{aligned}
$$

Now we integrate out the energy $\omega$ and interchange the order of integrations over momentum in the off-shell term,

$$
\begin{aligned}
E & =\int \frac{d k}{(2 \pi)^{3}} \varepsilon_{k} f(k)+\left.\int \frac{d k}{(2 \pi)^{3}} \varepsilon_{k} f(k) \frac{\partial \sigma_{00}}{\partial \omega}\right|_{\omega=\epsilon_{k}} \\
& +c \int \frac{d p}{(2 \pi)^{3}}\left|t_{00}^{R}\left(\epsilon_{p}\right)\right|^{2} f(p) \\
& \times \int \frac{d k}{(2 \pi)^{3}} \operatorname{Re} \frac{1}{\left(\epsilon_{p}-\epsilon_{k}+i 0\right)^{2}} .
\end{aligned}
$$

Similarly as in the case of the electron density, we join the second and the third terms, evaluate the integration over momentum $k$, and apply the derived optical theorem (72) to express the local energy in terms of the collision delay

$$
E=\int \frac{d k}{(2 \pi)^{3}} \varepsilon_{k} f(k)\left(1+\frac{\Delta_{t}}{\tau}\right) .
$$

[In notation of Paper I, Eq. (I-42), the quasiparticle energy does not include the potential $\phi$ that is in (I-42) explicitly added.] Thermodynamical consistency of the energy conservation and correlated density is shown in Appendix C of Paper I.

The BE (11) with subsidiary relations (103), (105) and (109) form a basic set of equations that cover most of traditional applications of the BE. In all these equations, the virial corrections can be included with help of collision delay. We remind that this simplicity follows in part from the simplicity of the scattering by Koster-Slater impurities.

\section{SUMMARY}

The intuitive modification of the BE, Eq. (11), has been recovered from non-equilibrium statistics. To this end we have employed non-equilibrium Green's functions within which we made the quasiclassical limit and the limit of small scattering rates. These two limits are fully sufficient, i.e., no unjustified approximations need to be made. The non-local form of the scattering integral in the intuitive BE has been obtained by unification of nongradient and gradient contributions.

Single-electron observables as functionals of the quasiparticle distribution are provided by the reduced density matrix that in the limit of small scattering rates has form (92). It was shown that (92) is consistent with the transport equation (11) leading to the correct equation of continuity discussed already in Paper I. The density of energy, which does not belong to single-electron observables, has been treated separately.

Presented theory has four general features that can be transferred to more general models. First, one needs sufficiently complex self-energy, the recommended one is based on the T-matrix which guarantees number of identities related to the optical theorem. Second, for small scattering rate, one can use the procedure of Refs. 14, 29 to derive a quasiclassical transport equation for quasiparticles. Resulting transport equation includes the quasiparticle and the virial corrections. The virial corrections are, however, in a form of gradient contributions to the scattering integral. Third, the virial corrections are rearranged to the semi-classical form when one recollects non-gradient and gradient terms on the scattering integral using logarithmic derivatives. Fourth, all logarithmic derivatives should be defined from the T-matrix, i.e., from the scattering phase shift. These logarithmic derivatives have natural interpretations like the collision delay discussed here. Authors of this paper have implemented presented approach to nuclear matter, see Ref. 35.

On the other hand, the discussed scattering on neutral impurities allows for a number of simplifications that are not available for more general scattering mechanisms. First, the self-energy and the transport vertex are independent of momentum which allows us to employ shifts in the complex plane with help of which one can conveniently express self-consistent quantities by their nonself-consistent counterparts. Second, the lack of momentum dependence leads to the lack of space non-localities of the scattering integral, therefore all virial corrections are described by the collision delay. Third, the momentum independence reflects that there is only a single scattering channel of s-symmetry. In general, different scattering channels have different collision delays, in the case of neutral impurities there is only a single collision delay what simplifies appreciably all related formulas. Fourth, due to the time-independence of the impurity potential, there are no gradient corrections to the scattering rate. Fifth, this time-independence also simplifies the energy conservation in collisions. Sixth, due to the absence of the dynamics of impurities, the virial corrections appear only in the scattering in. Here we have selected this simple scattering on neutral impurities to have free hands to focus on details of the method.

We have not discussed here consequences and interpretation of the virial corrections as it has been already done within the intuitive approach in Paper I. Our aim here is to confirm validity of this intuitive approach. 


\section{ACKNOWLEDGMENTS}

This work was supported from the Grant Agency of the Czech Republic under contract Nr. 202960098, the BMBF (Germany) under contract Nr. 06R0745(0), and the EC Human Capital and Mobility Programme.

\section{APPENDIX A: VIRIAL VERSUS QUASIPARTICLE CORRECTIONS IN EQUILIBRIUM}

The quasiparticle and the virial corrections enter the $\mathrm{BE}$ in different ways. From this point of view, they represent independent corrections that can be treated separately. On the other hand, for scattering by resonant levels, both corrections are of the same magnitude as it is demonstrated in Fig. 5 of Paper I. Striking similarity of their magnitudes raises a question up to what extent these two corrections are independent. To answer this question we briefly discuss equilibrium where one can benefit from well developed theory of quasipartiche and virial corrections based on Green's functions 36 38, 5

In equilibrium, the local density of electrons is given by the spectral function as

$$
n=\int \frac{d \omega}{2 \pi} f_{F D}(\omega) \int \frac{d k}{(2 \pi)^{3}} a(\omega, k) .
$$

In the limit of small scattering rates, the spectral function will be substituted from (16) with $\phi=0$ and lowest order approximation of the scattering rate, $\gamma=\gamma_{00}$.

In the limit of small scattering rates we can easily separate the quasiparticle contribution to the local density

$$
\begin{aligned}
n_{\text {pole }} & =\int \frac{d \omega}{2 \pi} f_{F D}(\omega) \int \frac{d k}{(2 \pi)^{3}} z 2 \pi \delta\left(\omega-\varepsilon_{k}\right) \\
& =\int \frac{d k}{(2 \pi)^{3}} z f(k),
\end{aligned}
$$

where $f(k) \equiv f_{F D}\left(\varepsilon_{k}\right)$, and the background contribution

$$
n_{\text {off }}=\int \frac{d \omega}{2 \pi} f_{F D}(\omega) \int \frac{d k}{(2 \pi)^{3}} \operatorname{Re} \frac{\gamma_{00}(\omega)}{\left(\omega-\epsilon_{k}+i 0\right)^{2}} .
$$

Using (58), $n_{\text {pole }}$ can be split into the free part (93) and wave-function renormalization reduction

$$
n_{\text {pole }}=n_{\text {free }}+n_{\mathrm{wfr}}
$$

where

$$
n_{\mathrm{wfr}}=\left.\int \frac{d k}{(2 \pi)^{3}} f(k) \frac{\partial \sigma_{00}}{\partial \omega}\right|_{\omega=\epsilon_{k}} .
$$

¿From decompositions of the density, $n=n_{\text {pole }}+n_{\text {off }}$ and $n=n_{\text {free }}+n_{\text {corr }}$, and $(\mathrm{A} 4$ the correlated density results as a sum of the off-pole and wave-function renormalization parts

$$
n_{\mathrm{corr}}=n_{\mathrm{off}}+n_{\mathrm{wfr}} .
$$

Using the Kramers-Krönig relation for the real part of the self-energy

$$
\sigma_{00}(\omega)=\operatorname{Re} \int \frac{d E}{2 \pi} \frac{1}{E-\omega+i 0},
$$

in ( $\mathrm{A} 5$, , one finds that the correlated density reads

$$
n_{\text {corr }}=\int \frac{d \omega}{2 \pi} \frac{d k}{(2 \pi)^{3}}\left(f_{F D}(\omega)-f(k)\right) \operatorname{Re} \frac{\gamma_{00}(\omega)}{\left(\omega-\epsilon_{k}+i 0\right)^{2}},
$$

where the terms weighted by $f_{F D}(\omega)$ and $f(k)$ results from $n_{\text {off }}$ and $n_{\mathrm{wfr}}$, respectively. Apparently, there is a partial compensation of these contributions to $n_{\text {corr }}$.

The quantum-mechanical expression (A8) can be also given the form of the semi-classical formula 102). To this end we reorganize $n_{\text {off }}$ starting from (A3),

$$
\begin{aligned}
n_{\text {off }} & =\int \frac{d \omega}{2 \pi} f_{F D}(\omega) \gamma_{00}(\omega) \operatorname{Re} \int \frac{d k}{(2 \pi)^{3}} \frac{1}{\left(\omega-\epsilon_{k}+i 0\right)^{2}} \\
& =-\int \frac{d \omega}{2 \pi} f_{F D}(\omega) \gamma_{00}(\omega) \frac{\partial}{\partial \omega} \operatorname{Re} \int \frac{d k}{(2 \pi)^{3}} \frac{1}{\omega-\epsilon_{k}+i 0} \\
& =-\int \frac{d \omega}{2 \pi} f_{F D}(\omega) \gamma_{00}(\omega) \frac{\partial \tilde{g}_{00}}{\partial \omega} .
\end{aligned}
$$

In the last line we have used that the integral over momentum above defines a local element of Green's function.

To evaluate $\gamma_{00}$, we use

$$
\gamma_{00}(\omega)=c(-2) \operatorname{Im} t_{00}^{R}(\omega),
$$

and the optical theorem [that follows from non-selfconsistent form of (41)]

$$
\operatorname{Im} t_{00}^{R}=\left|t^{R}\right|_{00}^{2} \operatorname{Im} \tilde{g}_{00}^{R} .
$$

If we express the local density of states in terms of the momentum integration

$$
-2 \operatorname{Im} \tilde{g}_{00}^{R}(\omega)=\int \frac{d p}{(2 \pi)^{3}} 2 \pi \delta\left(\omega-\epsilon_{p}\right),
$$

the off-pole contribution can be expressed in terms of the quasiparticle distribution,

$$
\begin{aligned}
n_{\text {off }} & =-c \int \frac{d p}{(2 \pi)^{3}} \frac{d \omega}{2 \pi} f_{F D}(\omega)\left|t_{00}^{R}(\omega)\right|^{2} 2 \pi \delta\left(\omega-\epsilon_{p}\right) \frac{\partial \tilde{g}_{00}}{\partial \omega} \\
& =-\left.c \int \frac{d p}{(2 \pi)^{3}} f(p)\left|t_{00}^{R}\left(\epsilon_{p}\right)\right|^{2} \frac{\partial \tilde{g}_{00}}{\partial \omega}\right|_{\omega=\epsilon_{p}} . \quad(\mathrm{A} 13
\end{aligned}
$$

Finally, we substitute (A13) into (A6)

$$
n_{\text {corr }}=\int \frac{d p}{(2 \pi)^{3}} f(p)\left(\frac{\partial \sigma_{00}}{\partial \omega}-c\left|t_{00}^{R}\right|^{2} \frac{\partial \tilde{g}_{00}}{\partial \omega}\right)_{\omega=\epsilon_{p}} .
$$


Formula (A14) is identical to the semi-classical expression (102). To prove this claim we employ the derived optical theorem.

It is advantageous to start from (102). First, we reorganize the ratio of the collision delay given by (65) to the lifetime given by (59) as $\frac{1}{\tau}=2 c \operatorname{Im} t_{00}^{R}=i c\left(t_{00}^{R}-t_{00}^{A}\right)$ as

$$
\begin{aligned}
\frac{\Delta_{t}}{\tau} & =i c\left(t^{R}-t^{A}\right) \frac{1}{2 i}\left(\frac{1}{t_{00}^{R}} \frac{\partial t_{00}^{R}}{\partial \omega}-\frac{1}{t_{00}^{A}} \frac{\partial t_{00}^{A}}{\partial \omega}\right) \\
& =\frac{c}{2} \frac{\partial}{\partial \omega}\left(t_{00}^{R}+t_{00}^{A}\right)-\frac{c}{2}\left(\frac{t_{00}^{A}}{t_{00}^{R}} \frac{\partial t_{00}^{R}}{\partial \omega}+\frac{t_{00}^{R}}{t_{00}^{A}} \frac{\partial t_{00}^{A}}{\partial \omega}\right) \\
& =\frac{\partial \sigma_{00}}{\partial \omega}-\frac{c}{2}\left(\frac{t_{00}^{A}}{t_{00}^{R}} \frac{\partial t_{00}^{R}}{\partial \omega}+\frac{t_{00}^{R}}{t_{00}^{A}} \frac{\partial t_{00}^{A}}{\partial \omega}\right) .
\end{aligned}
$$

¿From a non-self-consistent form of (41) one finds

$$
\frac{\partial t_{00}^{R}}{\partial \omega}=t_{00}^{R} \frac{\partial \tilde{g}^{R}}{\partial \omega}
$$

which substituted into $\mathrm{A} 15$ provides the derived optical theorem

$$
\frac{\Delta_{t}}{\tau}=\frac{\partial \sigma_{00}}{\partial \omega}-c\left|t_{00}^{R}\right|^{2} \frac{\partial \tilde{g}_{00}}{\partial \omega} .
$$

The l.h.s is exactly the bracket in (A14). Thus the semi-classical formula (102) is equivalent to the quantummechanical formula (A14).

To summarize this appendix we would like to remind the most important point. Within Green's function, virial and quasiparticle corrections enter the density together in an unresolved form, but the optical theorem and the derived optical theorem can be used to separate them and express virial corrections in terms of the collision delay.

${ }^{1}$ V. Špička, P. Lipavský and K. Morawetz, Phys. Rev. B.

${ }^{2}$ R. F. Snider, J. Stat. Phys. 61, 443 (1990).

${ }^{3}$ R. F. Snider, J. Stat. Phys. 63, 707 (1991).

${ }^{4}$ F. Laloë and W. J. Mullin, J. Stat. Phys. 59, 725 (1990).

${ }^{5}$ L. P. Kadanoff and G. Baym, Quantum Statistical Mechanics (Benjamin, New York, 1962).

${ }^{6}$ G. M. Eliashberg, Zh. Exp. Teor. Fiz. 41, 1241 (1961);

${ }^{7}$ P. Nozieres and J. M. Luttinger, Phys. Rev. 127, 1423 (1962).

8 J. M. Luttinger and P. Nozieres, Phys. Rev. 127, 1431 (1962).

${ }^{9}$ R. E. Prange and L. P. Kadanoff, Phys. Rev. 134, A566 (1964).

10 R. E. Prange and A. Sachs, Phys. Rev. 158672 (1967).

${ }^{11}$ P. Danielewicz, Ann. Phys. (N.Y.) 152, 305 (1984).

${ }^{12}$ W. Botermans and R. Malfliet, Phys. Rep. 198, 115 (1990).

13 J. Rammer and H. Smith, Rev. Mod. Phys. 58, 323 (1986).

${ }^{14}$ V. Špička and P. Lipavský, Phys. Rev. B 52, 14615 (1995).
${ }^{15}$ K. Morawetz and G. Röpke, Phys. Rev. E 51, 4246 (1995).

16 J. R. Barker, J. Phys. C 6, 2663 (1973).

17 J. R. Barker and D. K. Ferry, Phys. Rev. Lett. 42, 1779 (1979).

${ }^{18}$ I. B. Levinson, Zh. Eksp. Teor. Fiz. 57, 660 (1969) [Sov. Phys.-JETP 30, 362 (1970)].

${ }^{19}$ D. Calecki and N. Pottier, J. Phys. (Paris) Colloq. 42 C7271 (1981).

${ }^{20}$ J. R. Barker, J. Phys. (Paris) Colloq. 42 C7-245 (1981).

${ }^{21}$ A. P. Jauho and J. W. Wilkins, Phys. Rev. Lett. 49, 762 (1982).

${ }^{22}$ A. P. Jauho and J. W. Wilkins, Phys. Rev. B 29, 1919 (1984).

23 S. K. Sarker, Phys. Rev. B 32, 743 (1985).

${ }^{24}$ P. Lipavský, V. Špička and B. Velický, Phys. Rev. B 34, 6933 (1986).

${ }^{25}$ F. S. Khan, J. D. Davies and J. W. Wilkins, Phys. Rev. B 36, 2578 (1987).

${ }^{26}$ D. C. Langreth and J. W. Wilkins, Phys. Rev. B 6, 3189 (1972).

27 P. Lipavský, F. S. Khan and J. W. Wilkins, Phys. Rev. B 43, 6665 (1991).

${ }^{28}$ V. M. Kolomiets and V. A. Plyuiko, Physics of Atomic Nuclei 57, 931 (1994) [Yad. Fiz. 57, 992 (1994)].

${ }^{29}$ V. Špička and P. Lipavský, Phys. Rev. Lett 73, 3439 (1994).

30 P. Lipavský, F. S. Khan, A. Kalvová, and J. W. Wilkins, Phys. Rev. B 43, 6650 (1991).

${ }^{31}$ G. F. Koster and J. C. Slater, Phys. Rev. 95, 1167 (1954).

32 G. F. Koster and J. C. Slater, Phys. Rev. 96, 1208 (1954).

33 B. Velický, S. Kirkpatrick, and H. Ehrenreich, Phys. Rev. 175, 747 (1968).

34 J. E. G. Farina, in The International Encyclopedia of Physical Chemistry and Chemical Physics, edited by R. McWeeny (Pergamon, Oxford, 1983), Vol 4.

${ }^{35}$ V. Špička, P. Lipavský and K. Morawetz, to be published in Phys. Rev. Lett.

${ }^{36}$ D. Kremp, W. D. Kraeft, and A. D. J. Lambert, Physica A 127, 72 (1984).

${ }^{37}$ R. Zimmermann and H. Stolz, Phys. Status Solidi B 131, 151 (1985).

${ }^{38}$ M. Schmidt, G. Röpke, and H. Schulz, Ann. Phys. (NY) 202, 57 (1990). 